\title{
No Place Like Home? Disentangling Preferences for HIV Testing Locations and Services Among Men Who Have Sex with Men in China
}

\author{
Stephen W. Pan ${ }^{1,2} \cdot$ Maya Durvasula ${ }^{3}$. Jason J. Ong ${ }^{4,5} \cdot$ Chuncheng Liu ${ }^{2,6} \cdot$ Weiming Tang $^{2,7} \cdot$ Hongyun Fu $^{8}$. \\ Chongyi Wei $^{9} \cdot$ Cheng Wang $^{10} \cdot$ Fern Terris-Prestholt ${ }^{11}$. Joseph D. Tucker ${ }^{2,4,7}$
}

Published online: 18 December 2018

(c) The Author(s) 2018

\begin{abstract}
In China, some health departments and gay community-based organizations have begun to offer home-based HIV testing kits in order to augment test uptake among men who have sex with men (MSM). However, HIV test preferences and motivations for home-testing among MSM in China are not well understood. The HIV testing preferences of 803 MSM throughout China were evaluated using single-item assessment and a discrete choice experiment (DCE). In both the single-item assessment and DCE, participants expressed strong preference for free and anonymous testing by health professionals. Both approaches also indicated that naïve testers most prefer home testing. However, among previous testers, the single-item assessment indicated that "home" was the most preferred testing location (vs. hospital or clinic), while the DCE indicated that "home" was the least preferred testing location after controlling for anonymity. HIV home-testing may have limited appeal to previously tested Chinese MSM if anonymity is not maintained.
\end{abstract}

Keywords Choice analysis $\cdot$ Stated choice $\cdot$ Stated preference $\cdot$ Gay $\cdot$ Asia $\cdot$ Patient centered

\section{Introduction}

China has one of the world's highest reported HIV incidence rates among men who have sex with men (MSM) [1]. Moreover, rates of new infections among MSM in China are rising nationwide, having increased from 3.24 infections $/ 100$ person-years (PY) in 2005-2008 to 5.50 infections/100 PY

Electronic supplementary material The online version of this article (https://doi.org/10.1007/s10461-018-2366-0) contains supplementary material, which is available to authorized users.

Stephen W. Pan

stephen.pan@liverpool.ac.uk

1 Department of Health and Environmental Sciences, Xi' an Jiaotong-Liverpool University, 111 Ren'ai Road, Suzhou Dushu Lake Higher Education Town, Suzhou 215123, Jiangsu Province, China

2 UNC-Project China, Guangzhou, China

3 Department of Economics, Duke University, Durham, NC, USA

4 Faculty of Infectious and Tropical Diseases, London School of Hygiene and Tropical Medicine, London, UK

5 Central Clinical School, Monash University, Clayton, VIC, Australia in 2012-2014 [2]. One critical factor contributing to ongoing HIV transmission among MSM is delayed diagnosis of infection [1].

Early HIV diagnosis facilitates earlier initiation of antiretroviral therapy, which in turn ensures better clinical outcomes among people living with HIV and decreases the likelihood of secondary transmissions [3]. However, despite intense efforts to promote routine HIV testing, HIV testing rates among MSM in China remain low. According to a 2015 national online survey, $46 \%$ of MSM in China have never

6 Department of Sociology, University of California at San Diego, San Diego, CA, USA

7 School of Medicine, University of North Carolina at Chapel Hill, Chapel Hill, NC, USA

8 Eastern Virginia Medical School, Norfolk, VA, USA

9 School of Public Health, Rutgers University, New Brunswick, NJ, USA

10 Dermatology Hospital, Southern Medical University, Guangzhou, China

11 Department of Global Health and Development, Faculty of Public Health and Policy, London School of Hygiene and Tropical Medicine, London, UK 
received an HIV test [4]. One reason Chinese MSM are not testing more frequently may be because of dissatisfaction with current HIV testing service options [5, 6]. For example, MSM in China have indicated an affinity for testing in public hospitals and clinics [6], but concerns about confidentiality surrounding HIV or MSM status may discourage facilitybased testing. Previous studies have qualitatively identified an array of HIV testing service characteristics that Chinese MSM consider when deciding whether to test [6-11], but to date there has been little to no research that has quantitatively examined the relative importance of each HIV testing service characteristic. By elucidating how MSM in China weigh the importance of specific HIV testing service attributes, policymakers can better focus HIV testing service optimization on the attributes most likely to increase HIV test uptake.

Discrete choice experiments (DCE) are an established methodology to quantitatively estimate the preferences and relative influence of specific product or service attributes underpinning individual choice decisions [12, 13]. Based on random utility theory, DCEs presume that individuals make rational choices, that is, choices that maximize their satisfaction [14]. DCEs ask individuals to choose between hypothetical product or services options, and then individuals' stated choices are used to make inferences about their preferences [14]. These stated choices in DCEs enable researchers to quantitatively estimate the conscious or subconscious decision-making heuristics and "trade-off" thresholds that individuals use when making discrete choices [15]. Furthermore, by drawing inferences from individuals' stated choices, DCEs produce estimates of respondent preferences that are less subject to biases introduced when individuals are asked to explicitly report and assess the motivations underlying their choices [15]. Within the health services literature, DCEs have been used to elucidate patient preferences ranging from human papillomavirus vaccination among adolescent females [16], to treatment of osteoarthritis among older adults [17], and linkage to HIV care services among the general population [18]. However, few DCEs have examined HIV testing preferences in low or middle income countries (LMIC) [19-23] or among MSM.

In response to this limited understanding of HIV testing preferences among MSM in LMIC, we conducted a DCE to identify potential drivers of HIV testing decisions among MSM in China.

\section{Methods}

\section{Study Design}

From June 2016 to January 2017, the DCE was conducted among MSM by social media throughout China in three stages: (1) Identification of HIV testing service attributes and levels, (2) Generation of the DCE d-efficient design matrix, and (3) Implementation of the DCE.

\section{Identification of HIV Testing Service Attributes and Levels}

To identify HIV testing service attributes that influence HIV testing decisions among Chinese MSM, we performed a literature review and conducted five focus group discussions each with 4-6 self-identified MSM over 16 years old in Guangzhou, China from June to July $2016(n=24)$. The eligibility criteria for focus group participants was being a person over 16 years old who was born male and has ever had sex with another man. Focus group participants were recruited on social media by a local gay community-based organization and were asked to discuss their decisionmaking process and service considerations when deciding whether or not to test for HIV [24]. Focus group discussions were moderated by a self-identified gay Chinese MSM, verbally recorded, transcribed, translated from Chinese to English, and analyzed thematically and iteratively [25]. Focus group participants were provided an honorarium of \$15 USD.

The average age of focus group participants was 26.5 years (StdD: 6.3) and the majority of participants had previously received an HIV test (23/24, 95.8\%). Additional sociodemographic characteristics of focus group participants are presented in Supplementary Table S1. Results from focus group discussions revealed seven key HIV testing service attributes and 19 associated levels, which were used to develop DCE choice sets (Fig. 1). Notably, focus group participants consistently expressed that availability of HIV care services to link to was not a consideration when deciding whether or not to test.

Once HIV testing service attributes and levels were identified, we developed pictorial representations of each HIV testing attribute level that would be used in the DCE (e.g., label and picture of a hospital to represent hospital as a hypothetical testing location). To ensure that the pictorial representations were clear and intuitive, focus group participants were asked to provide collective feedback on iterations of graphics representing HIV testing attribute levels, and graphics and accompanying text were modified accordingly.

\section{Development of the d-Efficient Design Matrix}

For the current study, we used a d-efficient design matrix [26] that entailed randomizing participants to one of ten blocks and asking each individual to complete six choice tasks (60 unique choice tasks). For each choice task, the participant was instructed to select one of three testing alternatives: testing scenario $\mathrm{A}$, testing scenario $\mathrm{B}$, and opt-out (i.e., do not test) (Fig. 2). The number of blocks and choice 


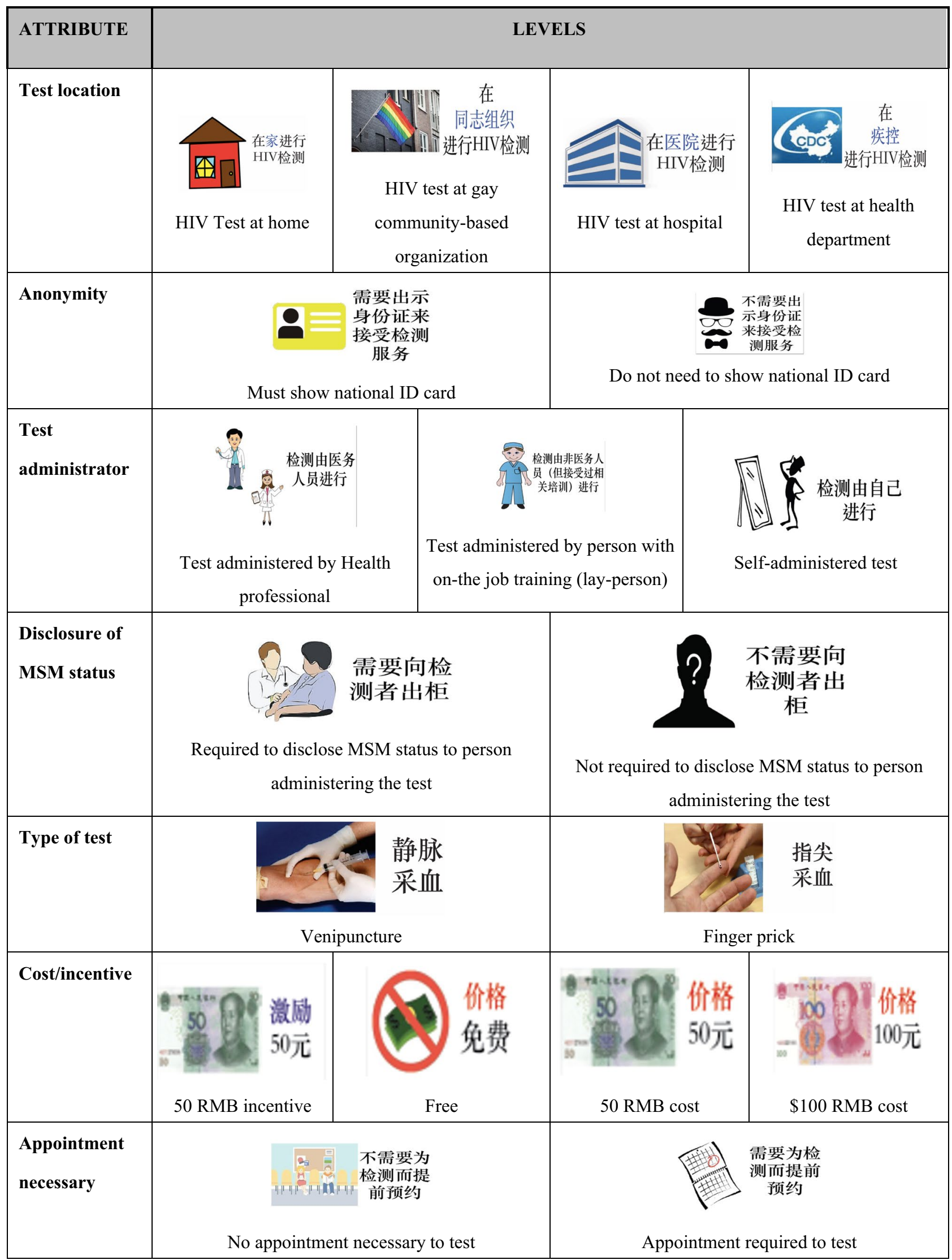

Fig. 1 Attributes and levels of the discrete choice experiment 


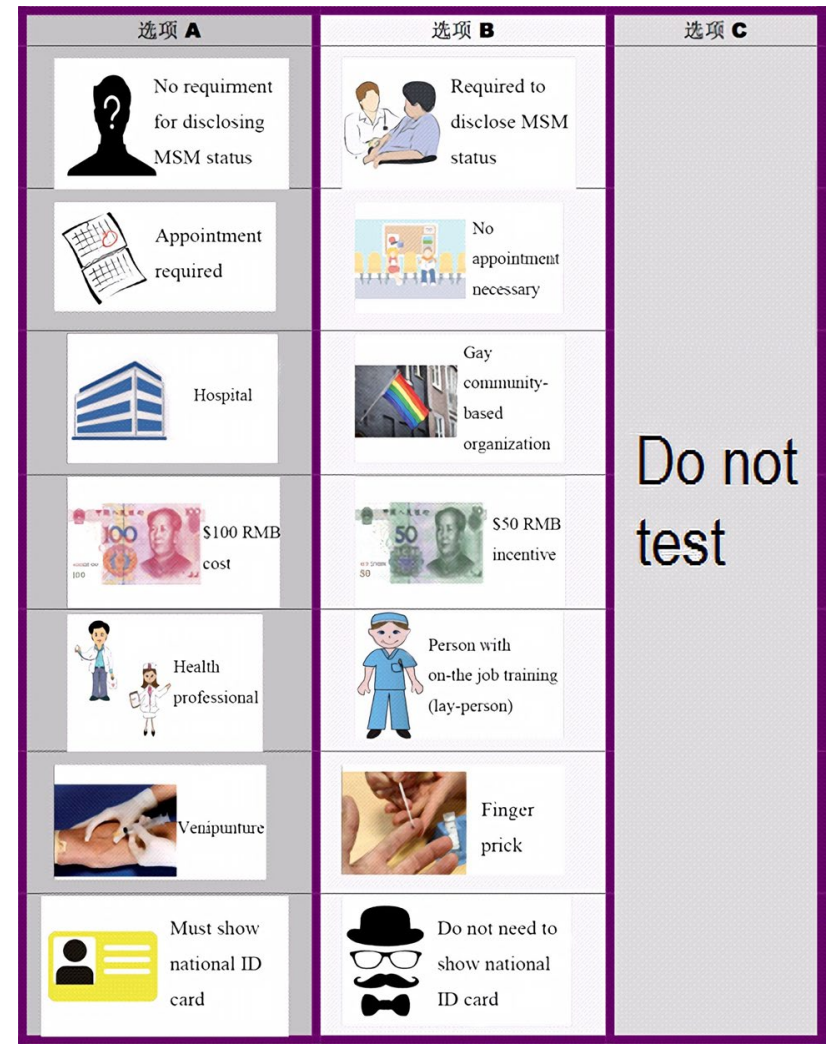

Fig. 2 Example of a choice set in the discrete choice experiment

tasks presented to each participant was based on considerations of potential survey fatigue and statistical efficiency of each design [27]. Block randomization, choice tasks, randomization of attribute ordering for each choice task, and d-efficiency statistics were produced in NGENE (ChoiceMetrics, 2014). No implausible testing scenarios were included (e.g., being required to disclose one's true name to the test administrators if self was the test administrator). In order to improve the precision of parameter estimates, the d-efficient design produced in NGENE used parameter estimate priors generated from an online pilot test conducted among 96 selfidentified MSM from two Chinese provinces.

\section{Implementation of the DCE}

The DCE was conducted online from January 8-31, 2017. Participants were recruited via multiple gay community based organizations and gay social networking portals throughout China. To participate in the study, individuals were required to self-report having been born biologically male, being over 16 years old (the legal age of adult consent), having ever had anal or oral sex with another man, and never having been diagnosed with HIV. To ensure ability to adequately assess preferences among subgroups of Chinese MSM, the following 1:1 ratio sampling quotas were imposed: income above and below $\$ 450$ USD per month, education above and below high school, and history of MSM behavior disclosure to healthcare worker (yes/no). Sub-analysis indicated that propensity to select the "opt-out" alternative did not significantly vary by education, income, or MSM disclosure status. Participants in the DCE pilot test survey received a $\$ 7.50$ USD phone credit honorarium delivered to their cell phone.

\section{Statistical Analyses}

Data analysis was conducted using three successive models: Multinomial logit (MNL), Mixed logit (MXL), and mixed logit with interaction terms (MXL-I). The MNL model included only main effects that provided overall averages of the study sample's HIV testing preferences. However, because the MNL approach does not account for unobserved preference heterogeneity between individuals or repeated observations collected from a single individual [28], we extended our analysis to include a MXL model. The MXL model only included main effects, all of which were set as random, assumed to be normally distributed, and modeled as non-linear, nominal categorical variables. Lastly, we used an MXL-I model that included parameter estimates for both attribute levels and interactions. In order to assess potential differences in testing preferences, the MXL-I model included two-way interaction terms between all design attributes and HIV testing history (ever received HIV test, yes vs. no). Model fit for the MXL and MXL-I models were compared using the log-likelihood ratio test. All analyses were conducted using the MLOGIT package in $\mathrm{R}$ and 1000 Halton draws. Effects and indicator coding were used for the design attributes and individual-specific characteristics, respectively. To facilitate clearer interpretation of relative satisfaction, design attribute coefficients were scaled from 0 to 10 .

The relative importance of each study attribute was calculated by dividing the range of parameter estimates for a given attribute, by the sum of parameter estimate ranges for all attributes [28]. Essentially, the relative importance for each attribute represents the proportion of DCE stated choices that were determined by the given attribute, excluding the influence of the opt-out selection and unmeasured factors (i.e., error terms). Confidence intervals for estimates of attribute relative importance were derived by calculating the standard error of each attribute's range using the following formula:

$\sqrt{ }[($ variance of attribute level $A)+($ variance of attribute level $B)$

$+(2 *$ covariance between attribute levels $A$ and $B)]$

[28]. Stratification was used to calculate the relative importance of testing attributes by HIV testing history (ever tested 
vs. never tested). Relative importance estimates and confidence intervals were based on MXL models.

\section{Ethical Review}

Study protocols were approved by the institutional review boards of the University of North Carolina at Chapel Hill (Study number 16-1860) and the Guangdong Provincial Dermatology Hospital. Each study participant was presented with an online consent form and provided informed consent prior to enrollment.

\section{Results}

In 23 days, the DCE survey link was clicked 3319 times. 2505 respondents failed to meet the study eligibility criteria or quit before eligibility could be established, and 11 respondents were removed after deduplication of identical phone numbers. The 803 eligible participants completed 4738 choice tasks. Alternative "A" (choice on the left side) and alternative "B" (choice on the right side) had comparable probabilities of being selected ( $47 \%$ vs. $45 \%$, respectively); the "opt-out" alternative was selected in $8 \%$ of all choice tasks.

\section{Participant Characteristics}

Participant characteristics are presented in Table 1. Overall, study participants were relatively young (median age: 24 years old), mostly single (86\%), mostly self-identified as gay $(78 \%)$, and most had previously tested for HIV (68\%). Distribution of education, income, and disclosure of MSM identity to health care providers aligned closely to the quota sampling scheme. Participants who never received an HIV

Table 1 Participant sociodemographics among MSM in China $(n=803)$

\begin{tabular}{|c|c|c|c|c|c|}
\hline & Code & $\begin{array}{l}\text { Total } \\
\text { n }(\%)\end{array}$ & $\begin{array}{l}\text { Among those who ever } \\
\text { received an HIV test (\%) }\end{array}$ & $\begin{array}{l}\text { Among those who never } \\
\text { received an HIV test (\%) }\end{array}$ & $\mathrm{p}$ value \\
\hline \multicolumn{6}{|l|}{ Age (years) } \\
\hline $1^{\text {st }}$ quantile $<21$ & 1 & $187(23)$ & (18) & $(34)$ & \multirow[t]{4}{*}{$<0.0001$} \\
\hline $2^{\text {nd }}$ quantile $21-23$ & 2 & $191(24)$ & (25) & $(21)$ & \\
\hline $3^{\text {rd }}$ quantile $24-28$ & 3 & $199(25)$ & (25) & (24) & \\
\hline $4^{\text {th }}$ quantile $>28$ & 4 & $226(28)$ & $(31)$ & (21) & \\
\hline \multicolumn{6}{|l|}{ Educational attainment } \\
\hline Elementary/middle & 1 & $137(17)$ & (15) & $(21)$ & \multirow[t]{4}{*}{0.02} \\
\hline High school & 2 & $287(36)$ & $(36)$ & $(36)$ & \\
\hline Vocational college & 3 & $129(16)$ & (15) & (19) & \\
\hline Four-year college and above & 4 & $250(31)$ & (34) & $(25)$ & \\
\hline \multicolumn{6}{|l|}{ Urban residency status } \\
\hline Official urban resident & 1 & $421(52)$ & $(55)$ & $(48)$ & \multirow[t]{2}{*}{0.08} \\
\hline Rural resident & 0 & $382(48)$ & $(45)$ & $(52)$ & \\
\hline \multicolumn{6}{|l|}{ Current marital status } \\
\hline Single & 1 & $689(86)$ & (84) & (90) & \multirow[t]{3}{*}{$<0.01$} \\
\hline Married & 2 & $77(10)$ & (10) & (9) & \\
\hline Separated/divorced/widowed & 3 & $37(5)$ & (6) & (1) & \\
\hline \multicolumn{6}{|l|}{ Sexual orientation } \\
\hline Gay & 1 & $623(78)$ & (80) & (73) & \multirow[t]{4}{*}{0.10} \\
\hline Heterosexual & 0 & $144(18)$ & (16) & (23) & \\
\hline Bisexual & 0 & $8(1)$ & (1) & (1) & \\
\hline Unsure & 0 & $28(3)$ & (4) & (3) & \\
\hline \multicolumn{6}{|l|}{ Income, USD/month } \\
\hline$<217$ & 1 & $177(22)$ & (19) & (28) & \multirow[t]{6}{*}{$<0.01$} \\
\hline $217-433$ & 2 & $246(31)$ & (31) & (30) & \\
\hline $434-724$ & 3 & $235(29)$ & (29) & (30) & \\
\hline $725-1159$ & 4 & $89(11)$ & (13) & (8) & \\
\hline$>1159$ & 4 & $56(7)$ & (8) & (4) & \\
\hline $\mathrm{n}$ & & 803 & 550 & 253 & \\
\hline
\end{tabular}


test were more likely to be less than 28 years old (79\% vs. $69 \%$ ), have only elementary or middle school education $(21 \%$ vs. $15 \%)$, and have income less than 217 USD per month ( $28 \%$ vs. $19 \%$ ) compared to men who had ever received an HIV test.

\section{Single-Item Assessment of Testing Preferences}

Single-item assessment of testing preferences (i.e., participants reporting their HIV testing preferences independently for each attribute) are presented in Table 2. Regarding test location, participants most preferred to test at home (34\%), followed by testing at gay community-based organizations (25\%), and local health departments (18\%). The most popular pricing models were free testing $(55 \%)$ and incentivized testing (24\%). Two out of three participants preferred walkin testing (66\%), while only one in five preferred appointment-based testing. The majority of individuals preferred to test anonymously (75\%), not be required to disclose their same-sex sexual activities (60\%), and to be tested by a trained health professional $(60 \%)$. Finger-prick testing was preferred over venous blood testing ( $48 \%$ vs. $27 \%$ ), but one in four participants was indifferent to the test type (25\%). Participants who had never tested before had a greater

Table 2 Single-item assessment of participants' HIV testing preferences among MSM in China $(\mathrm{n}=803)$

\begin{tabular}{|c|c|c|c|c|}
\hline & $\begin{array}{l}\text { Total } \\
\mathrm{n}(\%)\end{array}$ & $\begin{array}{l}\text { Among those who ever } \\
\text { received an HIV test (\%) }\end{array}$ & $\begin{array}{l}\text { Among those who never } \\
\text { received an HIV test }(\%)\end{array}$ & $\mathrm{p}$ value \\
\hline \multicolumn{5}{|l|}{ Test location ${ }^{\mathrm{a}}$} \\
\hline Home & $271(34 \%)$ & $(29)$ & $(45)$ & \multirow[t]{5}{*}{$<0.0001$} \\
\hline Gay community-based organization & $201(25 \%)$ & (26) & $(23)$ & \\
\hline Hospital & $75(9 \%)$ & (10) & (8) & \\
\hline Local health department & $144(18 \%)$ & $(21)$ & (11) & \\
\hline Indifferent & $109(14 \%)$ & (14) & (13) & \\
\hline \multicolumn{5}{|l|}{ Cost/incentive of test ${ }^{\mathrm{a}}$} \\
\hline$\$ 7.50$ USD incentive & $194(24 \%)$ & $(28)$ & (16) & \multirow[t]{5}{*}{$<0.001$} \\
\hline Free & $438(55 \%)$ & $(53)$ & $(58)$ & \\
\hline Pay $\$ 7.50$ USD & $34(4 \%)$ & (3) & $(6)$ & \\
\hline Pay $\$ 15$ USD & $44(6 \%)$ & (6) & (4) & \\
\hline Indifferent & $90(11 \%)$ & (9) & $(15)$ & \\
\hline \multicolumn{5}{|l|}{ Appointment versus walk-in testing ${ }^{\mathrm{a}}$} \\
\hline Walk-in & $532(66 \%)$ & $(67)$ & $(64)$ & \multirow[t]{3}{*}{0.12} \\
\hline Appointment necessary & $159(20 \%)$ & $(18)$ & $(24)$ & \\
\hline Indifferent & $112(14 \%)$ & $(15)$ & $(12)$ & \\
\hline \multicolumn{5}{|c|}{ Disclosure of same-sex sexual activities to test administrator ${ }^{\mathrm{a}}$} \\
\hline Not required to disclose & $483(60 \%)$ & $(60)$ & $(60)$ & \multirow[t]{3}{*}{0.27} \\
\hline Required to disclose & $189(24 \%)$ & $(22)$ & $(26)$ & \\
\hline Indifferent & $129(16 \%)$ & $(17)$ & $(13)$ & \\
\hline \multicolumn{5}{|l|}{ Test administrator ${ }^{\mathrm{a}}$} \\
\hline Trained health professional & $484(60 \%)$ & $(64)$ & $(52)$ & \multirow[t]{4}{*}{$<0.001$} \\
\hline $\begin{array}{l}\text { Individual with on the job training to admin- } \\
\text { ister test }\end{array}$ & $89(11 \%)$ & $(12)$ & (9) & \\
\hline Self-test & $164(20 \%)$ & $(15)$ & $(33)$ & \\
\hline Indifferent & $65(8 \%)$ & (9) & $(6)$ & \\
\hline \multicolumn{5}{|l|}{ Test type $^{\mathrm{a}}$} \\
\hline Finger prick & $387(48 \%)$ & $(46)$ & $(54)$ & \multirow[t]{3}{*}{$<0.001$} \\
\hline Venous & $217(27 \%)$ & $(31)$ & (18) & \\
\hline Indifferent & $198(25 \%)$ & $(23)$ & $(28)$ & \\
\hline \multicolumn{5}{|l|}{ Anonymity $^{\mathrm{a}}$} \\
\hline Not required to show national ID card & $604(75 \%)$ & (79) & $(67)$ & \multirow[t]{4}{*}{$<0.01$} \\
\hline Required to show national ID card & $132(16 \%)$ & (14) & $(22)$ & \\
\hline Indifferent & $66(8 \%)$ & (7) & (11) & \\
\hline $\mathrm{n}$ & 803 & 550 & 253 & \\
\hline
\end{tabular}

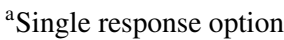


preference for testing at home ( $45 \%$ vs. $29 \%$ ), self-testing (33\% vs. $15 \%$ ), finger prick testing ( $54 \%$ vs. $46 \%$ ), and realname testing ( $22 \%$ vs. $14 \%$ ).

\section{Overall Design Attribute Effects}

\section{MNL Model}

Supplementary Table S2 shows participants' attributespecific HIV testing preferences based on results of the MNL analysis. In contrast to the single-item assessment, home was the least preferred testing location $(\beta=-0.10$, $\mathrm{p}<0.01)$. Notably, participants expressed slightly stronger preference for free testing over testing with monetary incentives ( $\beta=0.32$ vs. $\beta=0.23$ ). Preference ranking for all other parameter estimates were in the order as expected (e.g., stronger preference for cost of $\$ 7.50$ USD test vs. $\$ 15$ USD test).

\section{Model}

Table 3 shows results of the MXL analysis. Each attribute contained at least one level with statistically significant standard deviation estimates of the coefficient, thus implying substantial heterogeneity of preference weights across respondents for all attributes [28].
Table 3 HIV testing preferences of MSM in China (MXL model)

\begin{tabular}{|c|c|c|c|c|}
\hline Design attributes and levels & Coefficient & SE & StdD & SE \\
\hline \multicolumn{5}{|l|}{ Test location } \\
\hline Home & $-0.14^{*}$ & 0.05 & $0.75 * * *$ & 0.11 \\
\hline Community-based organization & 0.04 & 0.05 & $0.59 * * *$ & 0.12 \\
\hline Hospital & -0.05 & 0.05 & -0.01 & 0.65 \\
\hline Health department & $0.15^{* *}$ & 0.05 & 0.01 & 0.90 \\
\hline \multicolumn{5}{|l|}{ Identifier collected at test time } \\
\hline Must show ID card & $-0.33 * * *$ & 0.04 & $0.58 * * *$ & 0.07 \\
\hline Do not need to show ID card & $0.33 * * *$ & 0.04 & $0.61 * * *$ & 0.07 \\
\hline \multicolumn{5}{|l|}{ Test administrator } \\
\hline Self-test & -0.18 & 0.19 & $0.98 * * *$ & 0.12 \\
\hline Person with on-the job training & $-0.24 *$ & 0.10 & $-0.25^{*}$ & 0.12 \\
\hline Health professional & $0.41 * * *$ & 0.11 & 0.10 & 0.31 \\
\hline \multicolumn{5}{|l|}{ MSM identity disclosure } \\
\hline Required to disclose MSM activity & $-0.13 * * *$ & 0.03 & $0.46^{* * *}$ & 0.07 \\
\hline NOT required to disclose MSM activity & $0.13 * * *$ & 0.03 & $0.43 * * *$ & 0.07 \\
\hline \multicolumn{5}{|l|}{ Type of test } \\
\hline Venipuncture & 0.06 & 0.03 & $0.38 * * *$ & 0.08 \\
\hline Finger prick & -0.06 & 0.03 & $0.31 * * *$ & 0.09 \\
\hline \multicolumn{5}{|l|}{ Cost/incentive } \\
\hline 7.50 USD incentive & $0.31 * * *$ & 0.05 & $0.40 * *$ & 0.14 \\
\hline Free & $0.49 * * *$ & 0.06 & $0.66^{* * *}$ & 0.12 \\
\hline 7.50 USD cost & $-0.22 * * *$ & 0.06 & -0.04 & 0.47 \\
\hline 15 USD cost & $-0.59 * * *$ & 0.06 & 0.01 & 0.52 \\
\hline \multicolumn{5}{|l|}{ Scheduling } \\
\hline Walk-in & 0.06 & 0.03 & 0.14 & 0.17 \\
\hline Appointment necessary & -0.06 & 0.03 & $0.22 *$ & 0.11 \\
\hline \multicolumn{5}{|l|}{ Nonrandom parameter } \\
\hline Opt-out ASC & $-0.89 * * *$ & 0.05 & & \\
\hline \multicolumn{5}{|l|}{ Model fit statistics } \\
\hline Number of individuals & 803 & & & \\
\hline Number of completed choice sets & 4738 & & & \\
\hline Log-likelihood function & -4075.5 & & & \\
\hline
\end{tabular}

$* \mathrm{p}<0.05 ; * * \mathrm{p}<0.01 ; * * * \mathrm{p}<0.001$

$S t d D$ Standard deviation, $S E$ standard error, $A S C$ alternative-specific constant 


\section{MXL-I Model}

Table 4 shows results of the MXL-I analysis. The MXL-I model extends the MXL model to explore preference heterogeneity by testing experience. The log-likelihood ratio test indicated that the MXL-I model fit significantly better than the MXL model ( $\left.p<0.001, \chi^{2}=42.3,13 \mathrm{DF}\right)$. The MXL-I analysis indicated that preference for testing at home was significantly stronger among test-naïve men, compared to previous testers $(\beta=-0.58, \mathrm{p}<0.001)$. Participants with testing experience expressed significantly stronger preference for testing at the health department $(\beta=0.41$, $\mathrm{p}<0.001)$. In addition, naïve testers were significantly more likely to choose the opt-out choice, compared to men with testing experience $(\beta=0.23, \mathrm{p}<0.05)$.

A sensitivity analysis was conducted to examine how sociodemographic may have influenced preferences of naïve and experienced testers. The sensitivity analysis entailed rerunning the MXL-I analysis among sub-samples stratified by age, sexual orientation, and income. Results of the sensitivity analysis indicated that naïve testers' stronger preference for home testing and weaker preference for testing at the health department (compared to experienced testers) was consistent within each stratum of age, sexual orientation, and income levels (results available upon request).

\section{Scaled HIV Testing Preferences}

Figure 3 illustrates scaled HIV testing preferences, enabling direct comparisons between testing levels and attributes. Larger values indicate stronger preference for a specific testing characteristic. Results showed that switching from real-name testing to anonymous testing was as influential on participants' stated preferences as changing from $\$ 7.50$ USD out-of-pocket testing to free testing, or changing the test administrator from a lay-person to a health professional (gain of approximately 6 points on rescaled scale).

\section{Relative Importance}

Table 5 presents the relative importance of HIV testing attributes as percentages, stratified by HIV testing history. Overall, cost/incentive was the most important attribute $(34.0 \%, 95 \%$ CI 31.2-36.7\%), followed by anonymity $(20.8 \%, 95 \%$ CI $18.1-23.3 \%)$, and test administrator $(20.4 \%, 95 \%$ CI $7.0-30.4 \%)$. Testing location was of modest importance $(9.2 \%, 95 \%$ CI $5.7-12.4 \%)$, but disclosure of MSM activity (7.9\%, 95\% CI 5.3-10.4\%), test type $(3.9 \%, 95 \%$ CI $1.0-6.7 \%)$, and appointment scheduling $(3.7 \%, 95 \%$ CI $1.0-6.2 \%)$ were of limited importance. The relative importance of attributes was generally similar between participants with different testing histories.

\section{Discussion}

Today, MSM in China have increasingly diverse HIV testing options. Health professionals at hospitals and local health departments often provide free HIV screening tests for selfidentified MSM [29], though such testing conditions may not be appealing to MSM reluctant to disclose their MSM status to heterosexual individuals for fear of stigmatization and/or discrimination [30]. Larger cities often offer free and anonymous testing at gay community-based organizations staffed by MSM, but qualitative research suggests that some MSM may prefer testing at specialized hospitals staffed by formally trained health professionals [31]. Most recently, online sales of HIV self-testing kits now enable individuals to conduct self-administered home-based testing, an approach which has received monetary and explicit policy support from the Chinese government and local health departments [32].

However, in order to effectively deploy multi-pronged testing strategies that will increase test uptake, it is imperative to understand the component drivers of HIV testing preferences. Novel findings from this study are instructive for programs seeking to augment HIV testing among MSM in China, particularly government programs that may include promotion of home-based HIV testing kits. This study extends the literature of HIV testing preferences by using an experimental design to disentangle the motivations underpinning location and service preferences for HIV testing.

The DCE revealed several novel findings which had not been identified in previous HIV testing preference studies among Chinese MSM [6-11]. First, results indicated that MSM with different HIV testing histories have significantly different preferences for where to test. Among test-naïve men, home and the health department were the most and least favored testing locations, respectively. However, the exact opposite was true among men who had previously tested. One possible reason test-naïve men preferred to test at home is because they were expressing stated preferences for their hypothetical first HIV test, which may be perceived differently than subsequent tests. That is, men without any personal testing experience may feel more intimidated or apprehensive about testing at government health departments [6], and hence prefer to test in the comfort of their own home. Men with prior testing experience were likely more familiar with testing procedures, and therefore may have had fewer unanswered questions or concerns about testing at a health department. Alternatively, it is possible that naive testers' preference for home-testing was directly contributing to their lack of testing experience. That is, the absence of ideal home testing options may have been a key contributing factor for why naïve testers had not yet tested. 
Table 4 HIV testing preferences of MSM in China (MXL-I model)

\begin{tabular}{|c|c|c|c|c|}
\hline Design attributes and levels & Coefficient & SE & StdD & SE \\
\hline \multicolumn{5}{|l|}{ Test location } \\
\hline Home & $0.25 * *$ & 0.10 & $0.72 * * *$ & 0.11 \\
\hline Gay community-based organization (CBO) & 0.07 & 0.10 & $0.58 * * *$ & 0.12 \\
\hline Hospital & -0.13 & 0.09 & 0.02 & 0.77 \\
\hline Health department & $-0.19 *$ & 0.09 & & \\
\hline \multicolumn{5}{|l|}{ Identifier collected at test time } \\
\hline Must show ID card & $-0.28 * * *$ & 0.06 & $0.57 * * *$ & 0.07 \\
\hline Do not need to show ID card & $0.28 * * *$ & 0.06 & & \\
\hline \multicolumn{5}{|l|}{ Test administrator } \\
\hline Self-test & -0.21 & 0.33 & $0.97 * * *$ & 0.12 \\
\hline Person with on the job training & -0.25 & 0.17 & $-0.24^{*}$ & 0.12 \\
\hline Health professional & $0.46^{*}$ & 0.18 & & \\
\hline \multicolumn{5}{|l|}{ MSM identity disclosure } \\
\hline Required to disclose MSM activity & $-0.13^{*}$ & 0.05 & $0.47 * * *$ & 0.07 \\
\hline NOT required to disclose MSM activity & $0.13 *$ & 0.05 & & \\
\hline \multicolumn{5}{|l|}{ Type of test } \\
\hline Venipuncture & 0.04 & 0.06 & $0.37 * * *$ & 0.08 \\
\hline Finger prick & -0.04 & 0.06 & & \\
\hline \multicolumn{5}{|l|}{ Cost/incentive } \\
\hline 7.50 USD incentive & $0.22 *$ & 0.09 & $0.39 * *$ & 0.15 \\
\hline Free test & $0.47 * * *$ & 0.11 & $0.66^{* * *}$ & 0.12 \\
\hline 7.50 USD cost & -0.16 & 0.09 & -0.02 & 0.50 \\
\hline 15 USD cost & $-0.53 * * *$ & 0.09 & & \\
\hline \multicolumn{5}{|l|}{ Scheduling } \\
\hline Appointment necessary & -0.02 & 0.06 & 0.12 & 0.19 \\
\hline Walk-in & 0.02 & 0.06 & & \\
\hline \multicolumn{5}{|l|}{ Nonrandom parameter } \\
\hline Opt-out ASC & $-0.74 * * *$ & 0.09 & & \\
\hline \multicolumn{5}{|l|}{ Interaction terms } \\
\hline Ever had HIV test $*$ Home & $-0.58 * * *$ & 0.12 & & \\
\hline Ever had HIV test * Gay CBO & -0.03 & 0.12 & & \\
\hline Ever had HIV test * Hospital & 0.12 & 0.11 & & \\
\hline Ever had HIV test * Must show ID card & -0.07 & 0.08 & & \\
\hline Ever had HIV test * Self-test & 0.07 & 0.40 & & \\
\hline Ever had HIV test * Person with on the job training & 0.00 & 0.21 & & \\
\hline Ever had HIV test * Required to disclose MSM activity & 0.00 & 0.07 & & \\
\hline Ever had HIV test * Venipuncture & 0.02 & 0.07 & & \\
\hline Ever had HIV test $* 7.50$ USD incentive & 0.14 & 0.11 & & \\
\hline Ever had HIV test * Free test & 0.04 & 0.13 & & \\
\hline Ever had HIV test $* 7.50$ USD cost & -0.08 & 0.12 & & \\
\hline Ever had HIV test * Appointment necessary & 0.11 & 0.07 & & \\
\hline Ever had HIV test * optout & $-0.23 *$ & 0.11 & & \\
\hline \multicolumn{5}{|l|}{ Model fit statistics } \\
\hline Number of individuals & 803 & & & \\
\hline Number of observations & 4738 & & & \\
\hline Log-likelihood function & -4054.4 & & & \\
\hline
\end{tabular}

${ }^{*} \mathrm{p}<0.05 ; * * \mathrm{p}<0.01 ; * * * \mathrm{p}<0.001$

$S t d D$ Standard deviation, $S E$ standard error, $A S C$ alternative-specific constant 


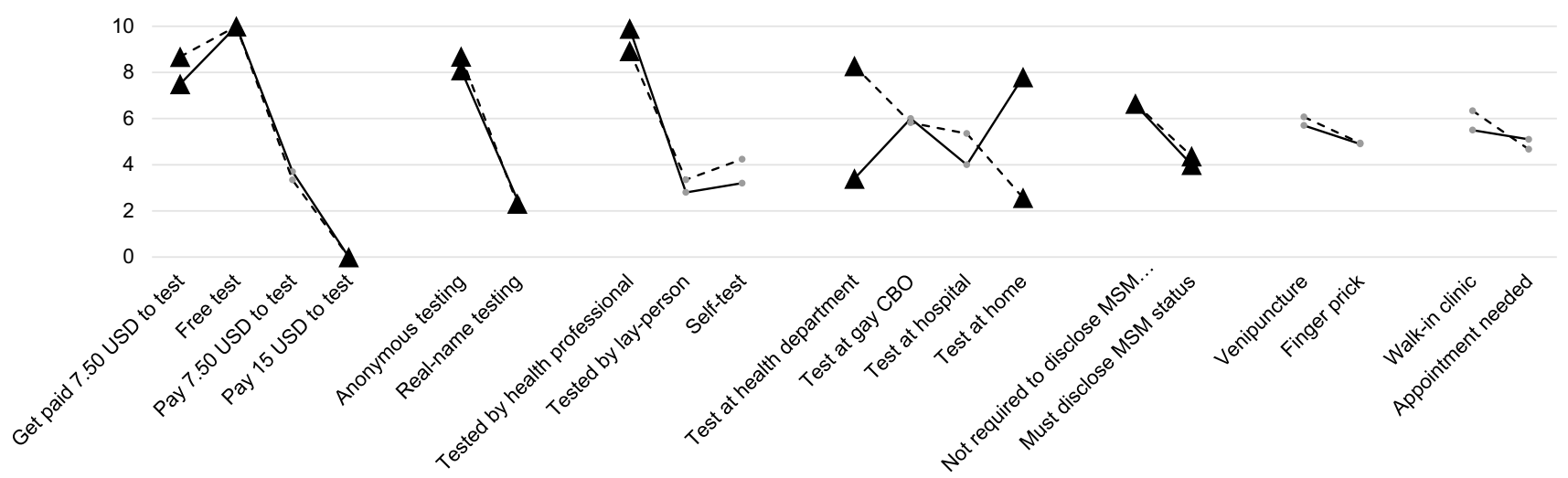

- Never tested - - - - Previously tested

$\Delta$ triangle denotes estimate is statistically significantly different from the mean preference

Fig. 3 Scaled estimates of HIV testing preferences among MSM in China, by testing history (MXL-I model) $(n=803)$

Table 5 Relative importance of HIV testing attributes among MSM in China (MXL models) $(\mathrm{n}=803)$

\begin{tabular}{llllllll}
\hline & Location & Anonymity & Test administrator & $\begin{array}{l}\text { Disclosure of } \\
\text { MSM activity }\end{array}$ & Test type & Cost/incentive & Appointment/walk-in \\
\hline Overall & $9.2 \%$ & $20.8 \%$ & $20.4 \%$ & $7.9 \%$ & $3.9 \%$ & $34.0 \%$ & $3.7 \%$ \\
& $(5.7-12.4 \%)$ & $(18.1-23.3 \%)$ & $(7.0-30.4 \%)$ & $(5.3-10.4 \%)$ & $(1.0-6.7 \%)$ & $(31.2-36.7 \%)$ & $(1.0-6.2 \%)$ \\
Ever tested & $17.8 \%$ & $19.3 \%$ & $17.0 \%$ & $6.7 \%$ & $3.6 \%$ & $30.8 \%$ & $5.0 \%$ \\
& $(14.3-21.0 \%)$ & $(16.3-22.0 \%)$ & $(3.1-27.5 \%)$ & $(3.9-9.3 \%)$ & $(0.4-6.5 \%)$ & $(27.3-33.8 \%)$ & $(2.2-7.7 \%)$ \\
Never tested & $13.9 \%$ & $18.5 \%$ & $22.1 \%$ & $7.7 \%$ & $2.6 \%$ & $33.5 \%$ & $1.6 \%$ \\
& $(8.0-19.2 \%)$ & $(14.2-22.4 \%)$ & $(15.3-27.9 \%)$ & $(3.5-11.5 \%)$ & $(2.2-6.9 \%)$ & $(28.0-38.3 \%)$ & $(0.0-5.7 \%)$ \\
\hline
\end{tabular}

Second, results from the DCE illuminated the potential limitations of measuring preferences by single-item assessment alone. According to the single-item assessment, home-testing was the most preferred testing location among previous testers. However, after accounting for factors such as anonymity and disclosure of MSM status, findings from the DCE indicated that home testing was the least preferred location among previous testers. This discrepancy between the single-item assessment and DCE results suggests that the popularity of home testing among previous testers was not due to any inherent qualities of the home environment, but rather because anonymity and confidentiality were relatively more assured by home testing. Future preference studies should be cautious about what types of conclusions can be inferred from single-item assessment methods.

Third, study results are among the first to rank the weighted importance of HIV testing attributes among MSM. Previous research has used qualitative data or single-item assessment to enumerate influential HIV testing service/product considerations among Chinese MSM [6-11], but methodological limitations of such study designs make it difficult to estimate measures of relative importance independently, which can lead to incorrect inferences. Using an experimental design that enabled estimates of testing attribute relative importance, we were able to identify which HIV testing service/product attributes exerted the strongest influence on stated preference, and potentially HIV test uptake. Specifically, cost, anonymity, and test administrator were the three most important testing attributes, collectively influencing $75 \%$ of all stated preferences. These findings are partially consistent with stated preference studies conducted in Africa [20, 22] and the United States [33], which also indicated cost as the most important attribute for patient HIV testing preferences.

However, a previous HIV testing stated preference study among MSM in the US did not identify anonymity as an especially important attribute [33]. This discrepancy may be partly explained by relatively stronger social stigmatization of homosexuality and same-sex sexual behaviors in China compared to the US [34]. Although same-sex sexual behaviors are legal and many sexual minority advocacy organizations operate openly [34], public expressions of sexual minority issues remain potentially subject to censure and censorship [35]. Given the social costs of disclosing MSM behaviors and identities in China, current government efforts 
to standardize mandatory real-name HIV testing [36] will almost certainly discourage HIV test uptake among MSM throughout China.

This study has several implications for HIV testing policy and research. First, findings indicate that home-based HIV self-test kits may have limited appeal to Chinese MSM with previous testing experience if anonymity is not maintained. It is now possible for individuals to purchase HIV self-test kits from the internet in China, but a substantial proportion of MSM may lack ability to routinely purchase and administer their own self-tests. As noted in this study and previous research from the US [33], cost is the single most important testing attribute for MSM when considering testing options. As government public health organizations in China begin to promote HIV self-testing kits to MSM alongside conventional facility-based testing services [37], it is incumbent for relevant policy makers to bear in mind that the appeal of home-based testing will be severely diminished if real-name testing is required. Simultaneously offering anonymous home-based and facilitybased testing options is likely to satisfy the preferences of naïve and previous testers alike.

Second, study findings suggest that small monetary incentives are unlikely to significantly increase test uptake among Chinese MSM, when compared to free testing. In both the single-item assessment and the DCE, participants expressed stronger preference for free testing, rather than testing with monetary incentives. From a classical economic perspective, this preference for free testing over incentivized testing may appear irrational and unexpected. However, this finding accords well with robust research from behavioral economics [38], which suggests that introducing monetary incentives can actually diminish the intrinsic motivation of getting tested for HIV (e.g., sense of satisfaction derived from taking care of one's health). Another possible explanation is that MSM may be distrustful of monetary incentives used to promote HIV testing. Campaigns and programs to boost HIV testing among MSM in China should consider non-monetary incentives, or approaches that can enhance intrinsic motivations.

\section{Limitations}

Several study limitations should be noted. First, generalizability may be limited because the HIV testing service attributes and levels used in the DCE were based on focus groups from a single, urban city in Southern China. It is possible that MSM in rural or Northern China may have qualitatively different HIV testing service considerations when deciding whether to test. Second, study findings may not be generalizable to completely off-line MSM in China, although by using sampling quotas, we were able to ensure greater sociodemographic representation. Third, measures of relative importance only reflect observed variation, and do not take into account the unobserved variation in stated preferences. Fourth, the stated preferences of HIV testing services may be distinct from HIV testing decisions in the real-world, and inferences about HIV testing decisions in the real-world must be made judiciously [12].

\section{Conclusion}

Supplementing conventional clinic-based testing with home-based HIV self-testing kits is a promising approach to increase HIV test uptake among MSM in China. However, findings from this study suggest that providing hometest kits to Chinese MSM may have limited impact on routine HIV test uptake unless testing is anonymous. Future studies should consider applying DCEs as a means of disentangling the motivations underpinning patient preferences at other stages of the HIV cascade of care.

Acknowledgements Many thanks to the study participants for sharing their time in order to make this study possible. Thanks also to Marco Boeri for providing technical advice on calculating standard errors of attribute relative importance.

Author Contributions JDT, FTP, and SWP conceptualized the study. SWP drafted the manuscript. SWP, MD, JJD, CL, WT, HF, CW, CW, FTP, and JDT provided substantial contribution to interpretation and revising the manuscript for important intellectual content. All authors approved the final version of the manuscript to be published and accept accountability for all aspects of the manuscript.

Funding This study was funded by the US National Institutes of Allergy and Infectious Diseases (NIAD1R01AI114310) and Fogarty International Center (R25TW009340).

\section{Compliance with Ethical Standards}

Conflict of interest SWP declares that he has no conflict of interest. MD declares that she has no conflict of interest. JJD declares that he has no conflict of interest. CL declares that he has no conflict of interest. WT declares that he has no conflict of interest. HF declares that she has no conflict of interest. CW declares that he has no conflict of interest. CW declares that he has no conflict of interest. FTP declares that she has no conflict of interest. JDT declares that he has no conflict of interest.

Ethical Approval All procedures performed in studies involving human participants were in accordance with the ethical standards of the institutional and/or national research committee and with the 1964 Helsinki declaration and its later amendments or comparable ethical standards.

Informed Consent Informed consent was obtained from all individual participants included in the study. 
Open Access This article is distributed under the terms of the Creative Commons Attribution 4.0 International License (http://creativeco mmons.org/licenses/by/4.0/), which permits unrestricted use, distribution, and reproduction in any medium, provided you give appropriate credit to the original author(s) and the source, provide a link to the Creative Commons license, and indicate if changes were made.

\section{References}

1. Beyrer C, Baral SD, Collins C, et al. The global response to HIV in men who have sex with men. Lancet. 2016;388(10040):198-206.

2. Zhang W, Xu J, Zou H, Zhang J, Wang N, Shang H. HIV incidence and associated risk factors in men who have sex with men in Mainland China: an updated systematic review and meta-analysis. Sex Health. 2017;13:373-82.

3. Joint United Nations Programme on HIV/AIDS. 90-90-90 An ambitious treatment target to help end the AIDS epidemic. 2014.

4. Zhang TP, Liu C, Han L, et al. Community engagement in sexual health and uptake of HIV testing and syphilis testing among MSM in China: a cross-sectional online survey. J Int AIDS Soc. 2017;20(1):1-10.

5. Lu H, Liu Y, Dahiya K, et al. Effectiveness of HIV risk reduction interventions among men who have sex with men in China: a systematic review and meta-analysis. PLoS ONE. 2013;8(8):e72747.

6. Han L, Wei C, Muessig KE, et al. HIV test uptake among MSM in China: implications for enhanced HIV test promotion campaigns among key populations. Glob Public Health. 2017;12(1):31-44.

7. Bien $\mathrm{CH}$, Muessig KE, Lee R, et al. HIV and syphilis testing preferences among men who have sex with men in South China: a qualitative analysis to inform sexual health services. PLoS ONE. 2015;10(4):e0124161

8. Wei C, Yan H, Yang C, et al. Accessing HIV testing and treatment among men who have sex with men in China: a qualitative study. AIDS Care. 2014;26(3):372-8.

9. Zhao Y, Zhang L, Zhang H, et al. HIV testing and preventive services accessibility among men who have sex with men at high risk of HIV infection in Beijing, China. Medicine. 2015;94(6):e534.

10. Song Y, Li X, Zhang L, et al. HIV-testing behavior among young migrant men who have sex with men (MSM) in Beijing, China. AIDS Care. 2011;23(2):179-86.

11. Li X, Lu H, Raymond HF, et al. Untested and undiagnosed: barriers to HIV testing among men who have sex with men, Beijing, China. Sex Transm Infect. 2012;88(3):187-93.

12. De Bekker-Grob EW, Ryan M, Gerard K. Discrete choice experiments in health economics: a review of the literature. Health Econ. 2012;21:145-72.

13. Lancaster KJ. A new approach to consumer theory. J Polit Econ. 1966;74(2):132-57.

14. Mcfadden D. Conditional logit analysis of qualitative choice behavior. In: Zarembka P, editor. Frontiers in econometrics. New York: Academic Press; 1973. p. 105-42.

15. Mangham LJ, Hanson K, McPake B. How to do (or not to do)... Designing a discrete choice experiment for application in a lowincome country. Health Policy Plan. 2009;24(2):151-8.

16. Hofman R, de Bekker-Grob EW, Raat H, Helmerhorst TJM, van Ballegooijen M, Korfage IJ. Parents' preferences for vaccinating daughters against human papillomavirus in the Netherlands: a discrete choice experiment. BMC Public Health. 2014;14(1):454.

17. Hauber AB, Arden NK, Mohamed AF, et al. A discrete-choice experiment of United Kingdom patients' willingness to risk adverse events for improved function and pain control in osteoarthritis. Osteoarthr Cartil. 2013;21(2):289-97.
18. D’Elbée M, Indravudh PP, Mwenge L, et al. Preferences for linkage to HIV care services following a reactive self-test: discrete choice experiments in Malawi and Zambia. AIDS. 2018;32(14):2043-9.

19. Ostermann J, Njau B, Mtuy T, Brown DS, Muhlbacher A, Thielman N. One size does not fit all: HIV testing preferences differ among high-risk groups in Northern Tanzania. AIDS Care. 2015;27(5):595-603.

20. Indravudh PP, Sibanda EL, Elbe M, et al. 'I will choose when to test, where I want to test': investigating young people' s preferences for HIV self-testing in Malawi and Zimbabwe. AIDS. 2017;31(Suppl 3):S203-12.

21. Ostermann J, Njau B, Brown DS, Mühlbacher A, Thielman N. Heterogeneous HIV testing preferences in an urban setting in tanzania: results from a discrete choice experiment. PLoS ONE. 2014;9(3):e92100.

22. Strauss M, George G, Lansdell E, et al. HIV testing preferences among long distance truck drivers in Kenya: a discrete choice experiment. AIDS Care. 2018;30(1):72-80.

23. Zanolini A, Chipungu J, Vinikoor MJ, et al. HIV self-testing in Lusaka Province, Zambia: acceptability, comprehension of testing instructions, and individual preferences for self-test kit distribution in a population-based sample of adolescents and adults. AIDS Res Hum Retrovir. 2017;34(3):254-60.

24. Michaels-igbokwe C, Lagarde M, Cairns J, Terris-prestholt F. Using decision mapping to inform the development of a stated choice survey to elicit youth preferences for sexual and reproductive health and HIV services in rural Malawi. Soc Sci Med. 2014;105:93-102.

25. Helter TM, Boehler CEH. Developing attributes for discrete choice experiments in health: a systematic literature review and case study of alcohol misuse interventions. J Subst Use. 2016;21(6):662-8.

26. ChoiceMetrics. Ngene 1.1.2 User manual \& reference guide. 2014;248.

27. Johnson FR, Lancsar E, Marshall D, et al. Constructing experimental designs for discrete-choice experiments: report of the ISPOR conjoint analysis experimental design good research practices task force. Value Heal. 2013;16(1):3-13.

28. Hauber AB, González JM, Groothuis-oudshoorn CGM, Prior T, Marshall DA, Cunningham C, et al. Statistical methods for the analysis of discrete choice experiments: a report of the ISPOR conjoint analysis good research practices task force. Value Heal. 2016;19(4):300-15.

29. Tucker JD, Wong FY, Nehl EJ, Zhang F. HIV testing and care systems focused on sexually transmitted HIV in China. Sex Transm Infect. 2012;88(2):116-9.

30. Watson J, Tang W, Pan S, et al. Out of the closet, into the clinic: opportunities for expanding men who have sex with men-competent services in China. Sex Transm Dis. 2018;45(8):527-33.

31. Liu Y, Sun X, Qian H-Z, et al. Qualitative assessment of barriers and facilitators of access to HIV testing among men who have sex with men in China. AIDS Patient Care STDS. 2015;29(9):481-9.

32. Tang $\mathrm{W}, \mathrm{Wu} \mathrm{D}$. Opportunities and challenges for HIV self-testing in China. Lancet HIV. 2018;3018(18):2017-8.

33. Lee SJ, Brooks R, Bolan RK, Flynn R. Assessing willingness to test for HIV among men who have sex with men using conjoint analysis, evidence for uptake of the FDA-approved at-home HIV test. AIDS Care. 2013;25(12):1592-8.

34. UNDP, USAID. Being LGBT in Asia: China Country report. 2014.

35. Palmer J. It's still (just about) OK to be gay in China [Internet]. Foreign Policy. 2018 [cited 2018 May 25]. p. 4. http://foreignpol icy.com/2018/04/17/its-still-just-about-ok-to-be-gay-in-china/. 
36. Centers for Disease Control and Prevention indicate support for real-name HIV testing [疾控中心表示支持艾滋病实名检测] [Internet]. legaldaily.com.cn. 2012. http://www.legaldaily.com. $\mathrm{cn} / \mathrm{bm} /$ content/2012-02/09/content_3340533.htm?node=20734. Accessed 5 Nov 2017.

37. Zhong F, Tang W, Cheng W, et al. Acceptability and feasibility of a social entrepreneurship testing model to promote HIV selftesting and linkage to care among men who have sex with men. HIV Med. 2017;18(5):376-82.
38. Frey BS, Jegen R. Motivation crowding theory a survey of empirical evidence. J Econ Surv. 2001;15(5):589-611.

Publisher's Note Springer Nature remains neutral with regard to jurisdictional claims in published maps and institutional affiliations. 